\title{
Hipoxemia inducida por el ejercicio en una paciente adulta con comunicación interauricular
}

\author{
Exercise-induced hypoxemia in an adult patient with an atrial septal defect \\ Carlos E. Vergara-Uzcátegui*, Pablo Salinas y Luis Nombela-Franco \\ Unidad de Cardiología Intervencionista, Hospital Clínico San Carlos. Madrid, España
}

La comunicación interauricular $(\mathrm{CIA})$ se presenta en 1.64 por 1,000 nacidos vivos y representa casi $10 \%$ de todas las cardiopatías congénitas ${ }^{1,2}$. Los adultos con CIA son casi siempre asintomáticos o señalan leve intolerancia a los esfuerzos, aunque puede demostrarse reducción de la función cardiopulmonar en ejercicio ${ }^{3}$.

\section{Caso clínico}

Mujer de 65 años con hallazgo de hipoxemia durante el estudio preoperatorio para colecistectomía programada tras lo cual se la deriva al servicio de cardiología. Tiene antecedente de ictus isquémico a los 56 años. Se hallaba en seguimiento previo en el servicio de neumología desde 2001 por hipoxemia, poliglobulia y disnea de esfuerzos, con espirometría normal y presión arterial de oxígeno $\left(\mathrm{pO}_{2}\right)$ de $49 \mathrm{mmHg}$. La tomografía computarizada torácica de alta resolución es normal. En 2011 se sometió a gammagrafía pulmonar con macroagregados de albúmina consistentes con cortocircuito de derecha a izquierda, pero abandonó el seguimiento.

La exploración física actual revela acropaquias, auscultación pulmonar normal, auscultación cardíaca con desdoblamiento y reforzamiento del segundo ruido en foco pulmonar. $\mathrm{pO}_{2}$ arterial de $46 \mathrm{mmHg}$ y saturación de $\mathrm{O}_{2}$ de $85 \%$. Los análisis muestran hemoglobina de $16.3 \mathrm{mg} / \mathrm{dl}$ y hematócrito de 50.4\%. El electrocardiograma delinea ritmo sinusal con signos de crecimiento biauricular, hemibloqueo anterior izquierdo y escasa progresión de R en V3-V6.

El ecocardiograma transtorácico (ETT) registró ventrículo izquierdo y derecho de tamaño y función normales, insuficiencia mitral y tricuspídea leve, presión sistólica de la arteria pulmonar normal y tabique interauricular (TIA) aneurísmico. La aurícula izquierda (Al) es de tamaño normal y la derecha (AD) está levemente dilatada. El ecocardiograma transesofágico (ETE) mostró aneurisma del TIA con CIA de tipo ostium secundum de $13 \times 8 \mathrm{~mm}$ en zona anterosuperior (Fig. 1A). El Doppler a color describe un cortocircuito bidireccional y abundante y paso temprano de burbujas a cavidades izquierdas sin maniobra de Valsalva, lo que podría explicar la hipoxemia de la paciente (Fig. 1B). Se realiza resonancia magnética cardíaca (Fig. 1 CyD) que muestra CIA amplia con cortocircuito bidireccional, ventrículo derecho (VD) de tamaño y función normales, y anatomía de las venas pulmonares normal con desembocadura en la Al. En el estudio de flujos se determina una relación $Q p / Q s$ de 0.8 , lo que sugiere cortocircuito predominante de derecha a izquierda. No hay dilatación de la arteria pulmonar.

Se realizó prueba de marcha de 6 minutos (6MWT) y se observa saturación inicial de $85 \%$, con disminución progresiva hasta $74 \%$ desde el minuto 4 . Luego de la prueba, la saturación final en reposo fue de $84 \%$ y la distancia recorrida de $306 \mathrm{~m}$.

\section{Correspondencia:}

${ }^{*}$ Carlos E. Vergara-Uzcátegui

E-mail: carting1@gmail.com
Fecha de recepción: 18-05-2020

Fecha de aceptación: 07-03-2021

DOI: 10.24875/ACM.20000225
Disponible en internet: 02-07-2021 Arch Cardiol Mex. 2021;91(3):375-378

www.archivoscardiologia.com

1405-9940 / @ 2021 Instituto Nacional de Cardiología Ignacio Chávez. Publicado por Permanyer. Este es un artículo open access bajo la licencia CC BY-NC-ND (http://creativecommons.org/licenses/by-nc-nd/4.0/). 


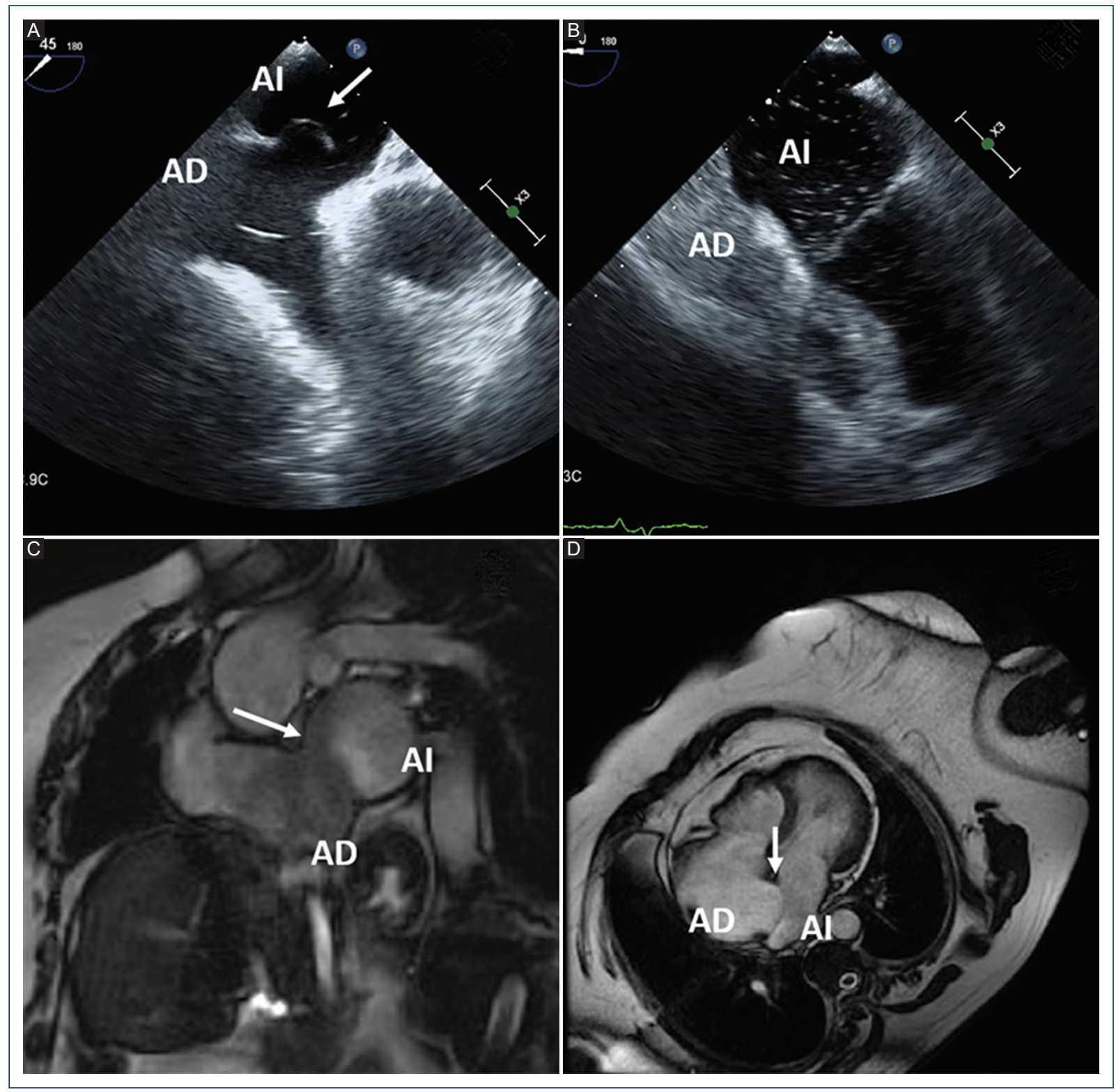

Figura 1. A: Ecocardiograma transesofágico a nivel medioesofágico a $45^{\circ}$ que muestra aneurisma del TIA (flecha) con CIA de tipo ostium secundum en zona anterosuperior. B: Vista de cuatro cámaras en la que se observa paso temprano de burbujas a las cavidades izquierdas. C: Resonancia magnética cardíaca en vista de eje corto que delinea el paso de contraste hacia la AI (flecha). D: Vista de cuatro cámaras que muestra TIA aneurísmico (flecha).

El cateterismo derecho no reveló salto oximétrico significativo en cavidades derechas, signo indicativo de cortocircuito neutro o de derecha a izquierda, presión capilar pulmonar y presión de aurícula derecha normales (10 y $8 \mathrm{mmHg}$, respectivamente). Gasometría arterial en decúbito supino de $84 \%$ y en bipedestación de $90 \%$ (leve mejoría de $6 \%$ que descarta platipnea-ortodesoxia). Se realizó cierre percutáneo de CIA con dispositivo Amplatzer Septal Occluder (St. Jude Medical $囚$ ) de 22 mm sin complicaciones. La $\mathrm{pO}_{2}$ con aire ambiente pasó de 49 a $89 \mathrm{mmHg}$ después del procedimiento.

En el seguimiento posterior al cierre, el 6MWT mostró saturación basal de 95\%, con disminución a $92 \%$ en el minuto 4 y saturación final en reposo de $96 \%$. Distancia recorrida de $405 \mathrm{~m}$. En el seguimiento a los 20 meses tras el procedimiento, la paciente ha mejorado la clase funcional (NYHA I) y tiene hemoglobina de $15.8 \mathrm{~g} / \mathrm{dl}$. En el ETE posterior al cierre se observa 


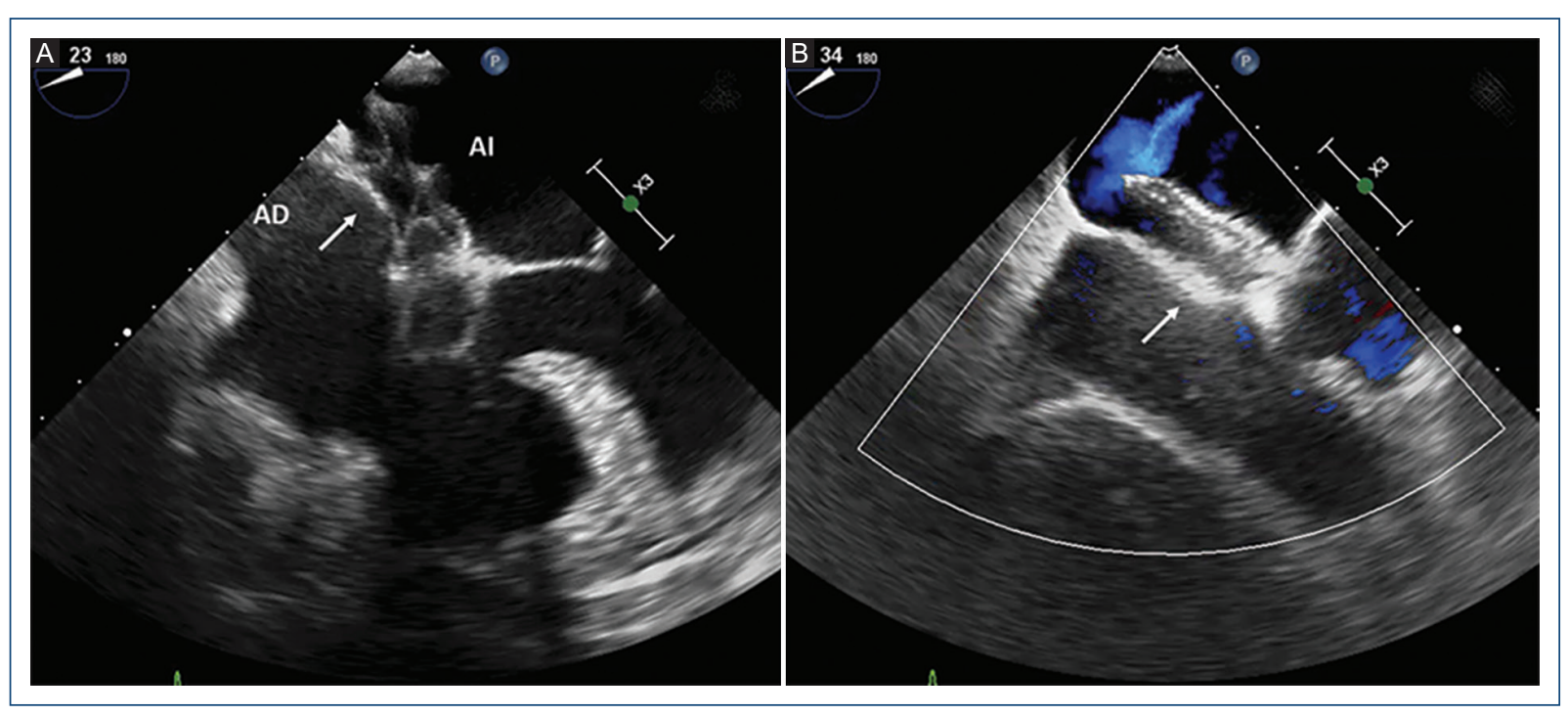

Figura 2. Ecocardiograma transesofágico posterior al cierre. A: Vista de cuatro cámaras. B: Vista a nivel medioesofágico a $34^{\circ}$ que muestra el dispositivo bien implantado sin cortocircuito residual.

dispositivo implantado normal en el TIA, sin cortocircuito residual (Fig. 2AyB).

\section{Discusión}

El cortocircuito de derecha a izquierda a través del TIA es una causa rara de hipoxemia inducida por el ejercicio 4 . Esta desaturación inducida por el ejercicio haría posible un cortocircuito de derecha a izquierda de manera similar a la platipnea-ortodesoxia, pero técnicamente distinta. Este mecanismo de desaturación en presencia de un agujero oval permeable (AOP)/CIA puede reflejar una desaturación arterial inducible y no un cortocircuito permanente, y es un fenómeno rara vez observado ${ }^{5}$. La hipoxemia resultante de un cortocircuito de derecha a izquierda es común en pacientes con CIA y resistencias vasculares pulmonares elevadas, pero infrecuente ante hipertensión pulmonar. Se reconoce en adultos de mediana edad con modificación de la orientación del TIA ${ }^{6}$.

Dos mecanismos se han postulado para explicar esta hipoxemia: la existencia de un gradiente de presión interauricular; y la distorsión anatómica de la relación cava inferior-TIA y defecto productor de un flujo preferencial de cava inferior hacia la $\mathrm{Al}^{6}$. En términos fisiológicos, la presión de la Al es de 5 a $8 \mathrm{mmHg}$ más alta que la presión en la $A D^{5}$. Se ha descrito un gradiente de presión sistólico entre AD-Al en pacientes con AOP/CIA sin hipertensión pulmonar en presencia de mixomas en $A D$, infarto del VD y ventilación mecánica, en particular en pacientes con presión positiva al final de la espiración elevada. Este gradiente puede aumentar por cambios posturales, inspiración, maniobras de Valsalva y disminución de la distensibilidad del VD o la $A D^{6}$. El ejercicio riguroso podría incrementar en grado suficiente el retorno venoso para generar un gradiente de presión e iniciar un cortocircuito transitorio ${ }^{5}$. En el plano anatómico, el flujo de sangre preferencial que proviene desde la vena cava superior o más a menudo desde la vena cava inferior hacia la Al por medio de una válvula de Eustaquio muy desarrollada podría también explicar el fenómeno del cortocircuito de derecha a izquierda y ocurrir en presencia de presiones normales en cavidades derechas, o en ausencia de gradiente de presión entre ambas aurículas ${ }^{6}$.

En esta paciente, el diagnóstico de una causa cardíaca que explicara la hipoxemia se retrasó debido a que no se identificó la CIA en ETT previos y la paciente dejó de asistir a las consultas. La decisión de cerrar una CIA de tamaño pequeño o moderado en adultos sin dilatación de cavidades derechas es controvertida; el escenario específico no está previsto en las guías y podría considerarse la indicación en caso de embolismo paradójico (antecedente de ictus en esta paciente). En el caso descrito se recomendó el cierre como tratamiento de la hipoxemia luego de reconocer el cortocircuito de derecha a izquierda y como prevención de posibles embolias paradójicas.

El 6MWT es una herramienta clínica simple y útil en la valoración del estado funcional; en pacientes muy sintomáticos, la correlación es buena con la prueba 
cardiopulmonar de ejercicio ${ }^{2}$. En esta paciente fue útil la prueba y mostró disminución de la saturación de oxígeno hasta de $11 \%$, con distancia recorrida de $306 \mathrm{~m}$ y clase funcional III de la NYHA.

La ecocardiografía con contraste es útil para excluir, en casos como éste, defectos del TIA4 . El ETE puede mostrar el cortocircuito de derecha a izquierda en un AOP o CIA mediante Doppler a color o después de la inyección intravenosa de contraste ${ }^{6}$ y además puede valorar distorsiones anatómicas, como válvula de Eustaquio prominente o desviación del tabique por dilatación aórtica.

En casos bien seleccionados, el cierre del defecto lleva a la resolución de la desaturación arterial y mejora la capacidad funcional en pacientes con desaturación inducida por el ejercicio ${ }^{5}$. En caso de duda se puede realizar una prueba de oclusión del defecto con balón, aunque se debe tener en cuenta que la posición anatómica en la sala de hemodinámica podría no ser representativa de la desaturación en un plano supino en los casos de platipnea-ortodesoxia. En esta paciente se realizó el cierre percutáneo con éxito y con mejoría inmediata de saturación, y durante los seguimientos se observaron una mejoría de la capacidad funcional, la ausencia de desaturación considerable en el 6MWT y el aumento de un $32 \%$ de la distancia recorrida. Aunque la valoración de la clase funcional puede ser subjetiva, la mejoría de la desaturación arterial parece ser un fenómeno común en los pacientes tratados ${ }^{5}$.

\section{Financiamiento}

Esta investigación no ha recibido ayuda específica proveniente de agencias de los sectores público o comercial ni de entidades sin ánimo de lucro.

\section{Conflicto de intereses}

Luis Nombela-Franco es proctor de Abbott Medical. No hay otros conflictos de intereses.

\section{Responsabilidades éticas}

Protección de personas y animales. Los autores declaran que para esta investigación no se han realizado experimentos en seres humanos ni en animales.

Confidencialidad de los datos. Los autores declaran que han seguido los protocolos de su centro de trabajo sobre la publicación de datos de pacientes.

Derecho a la privacidad y consentimiento informado. Los autores han obtenido el consentimiento informado de los pacientes o sujetos referidos en el artículo. Este documento obra en poder del autor de correspondencia.

\section{Bibliografía}

1. Supomo S, Darmawan $H$, Arjana AZ. Role of pulmonary hemodynamics in determining 6-minute walk test result in atrial septal defect: an observational study. J Cardiothorac Surg. 2018;13:51.

2. Wang, S, Pan, J, Xiao, B. Immediate and shortnterm effects of transcatheter device closure of large atrial septal defect in senior people. Congenital Heart Disease. 2019;14:939-44.

3. Khan AA, Tan JL, Li W. The impact of transcatheter atrial septal defect closure in the older population. J Am Coll Cardiol Intv. 2010;3:276-81.

4. Eckersley L, Clements B, Shipton S. Exercise-induced hypoxia secondary to an atrial septal defect and cor triatriatum dexter. Cardiology in the Young. 2016;26:793-5

5. Devendra GP, Rane AA, Krasuski RA. Provoked exercise desaturation in patent foramen ovale and impact of percutaneous closure. J Am Coll Cardiol Intv. 2012;5:416-9.

6. Godart F, Rey C, Prat A. Atrial right-to-left shunting causing severe hypoxaemia despite normal right-sided pressures. Report of 11 consecutive cases corrected by percutaneous closure. Eur Heart J. 2000; 21:483-9. 